\title{
A Compact High-Pass Filter Using Hybrid Microstrip/Nonuniform CPW with Dual-Mode Resonant Response
}

\author{
Hui Chen, ${ }^{1}$ Di Jiang, ${ }^{2}$ Ke-Song Chen, ${ }^{1}$ and Hong-Fei Zhao ${ }^{1}$ \\ ${ }^{1}$ School of Electronic Engineering, University of Electronic Science and Technology of China, Chengdu 611731, China \\ ${ }^{2}$ School of Communication and Information Engineering, University of Electronic Science and Technology of China, \\ Chengdu 611731, China
}

Correspondence should be addressed to Hui Chen; huichen@uestc.edu.cn

Received 1 April 2016; Revised 12 August 2016; Accepted 21 August 2016

Academic Editor: Paolo Baccarelli

Copyright (C) 2016 Hui Chen et al. This is an open access article distributed under the Creative Commons Attribution License, which permits unrestricted use, distribution, and reproduction in any medium, provided the original work is properly cited.

\begin{abstract}
A novel and miniature high-pass filter (HPF) based on a hybrid-coupled microstrip/nonuniform coplanar waveguide (CPW) resonator is proposed in this article, in which the designed CPW has exhibited a wideband dual-mode characteristic within the desired high-pass frequency range. The implemented filter consists of the top microstrip coupled patches and the bottom modified nonuniformly short-circuited CPW resonator. Simulated results from the electromagnetic (EM) analysis software and measured results from a vector network analyzer (VNA) show a good agreement. A designed and fabricated prototype filter having a 3 $\mathrm{dB}$ cutoff frequency $\left(f_{c}\right)$ of $5.78 \mathrm{GHz}$ has shown an ultrawide high-pass behavior, which exhibits the highest passband frequency exceeding $4.0 f_{c}$ under the minimum insertion loss (IL) $0.75 \mathrm{~dB}$. The printed circuit board (PCB) area of the filter is approximately $0.062 \lambda_{g} \times 0.093 \lambda_{g}$, where $\lambda_{g}$ is the guided wavelength at $f_{c}$.
\end{abstract}

\section{Introduction}

In $\mathrm{RF} /$ microwave wireless applications, the high-pass filters (HPFs) are important circuit elements, while the conventional procedures of implementing the HPFs often utilize the distributed units or quasi-lumped elements, which require grounded via-holes. As a result, these methods might lead to fabrication problems and also require large PCB sizes [1]. Recently, some new HPFs using various physical structures and design methods have been reported successively [27]. Among the newly published HPFs, the filters based on the various metamaterial concepts have been demonstrated in [2-5]. A new approach to designing a maximum flat Butterworth HPF, which transforms an open circuit series stub to short circuit shunt stub, has been extensively investigated in [6]. An elliptic-function response HPF based on SIR in coplanar waveguide (CPW) technology has also been researched in [7]. The above-overviewed high-pass frequency ranges of HPFs are basically up to $(2 \sim 3) f_{c}$.
Generally, the dual-mode (or multimode) resonant characteristics have been extensively applied to design UWB $\mathrm{BPF}$ and dual-band (or multiband) filters [8-13]. However, they are rarely used to implement the HPF. In this paper, a nonuniformly modified short-circuited $\mathrm{CPW}$ has been used to design the ultracompact HPF without any viahole, as the via-hole often leads to some fabrication problems. Different from CPW resonators discussed in [14, 15], the proposed CPW is based on the multisection stepped impedance structure, while a conventional and uniform CPW and a composite CPW using two $\lambda / 4 \mathrm{CPW}$ resonators and a short-circuited stub have been introduced in [14] and [15], respectively. The improved CPW resonator is obtained by etching slots in the ground plane of the two coupled microstrip rectangular patches. In this design, the nonuniform resonator has shown an ultrawide dual-mode frequency characteristic within the desired high-pass range. With the help of the two top microstrip parallel-coupled patches, the lower frequency attenuation for the band-stop 


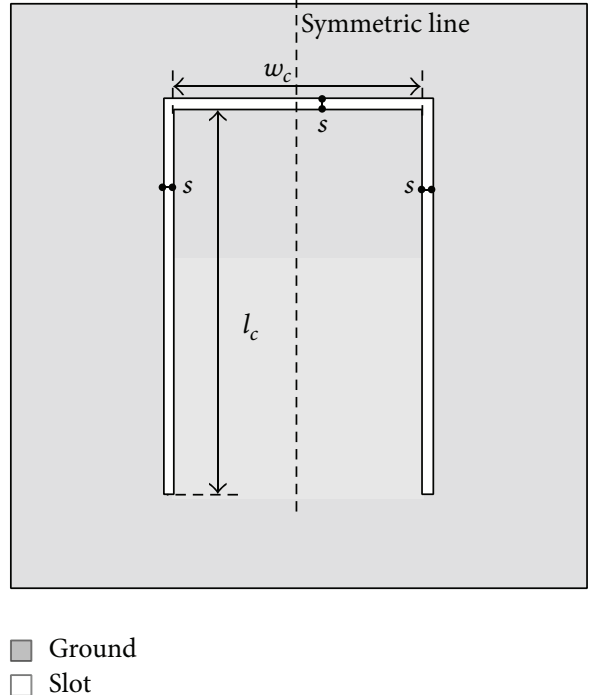

(a)
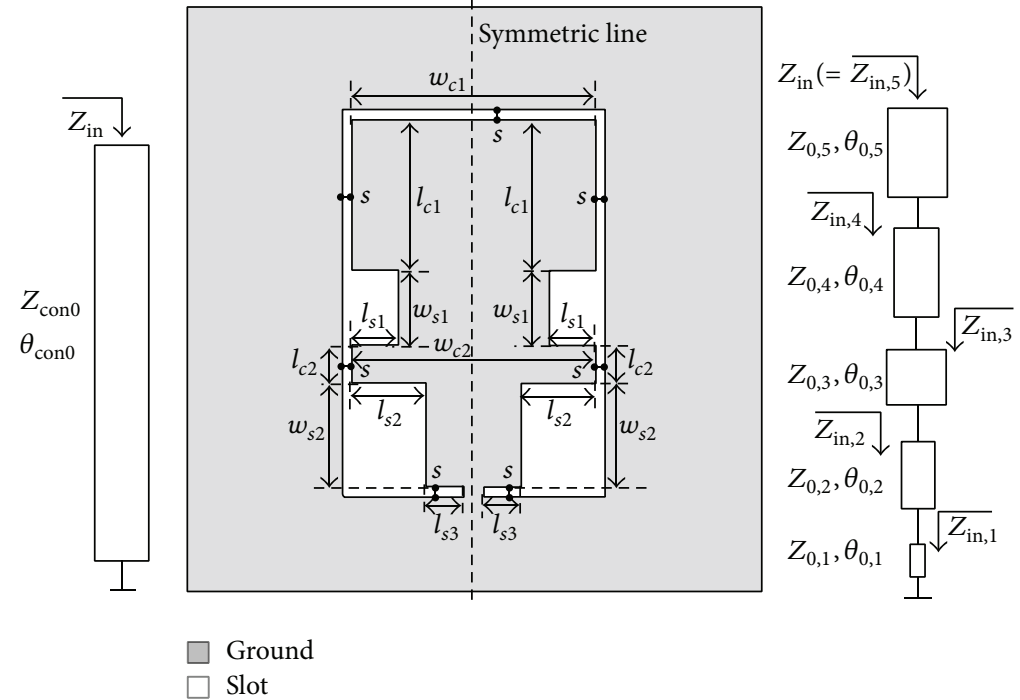

(b)

FIGURE 1: (a) Conventional short-circuited CPW resonator and its transmission-line equivalent circuit and (b) proposed nonuniform shortcircuited CPW resonator with dual-mode property and its transmission-line equivalent circuit.

and the upper passband transmission have been achieved. This excellent filtering circuit, having the high-pass frequency response exceeding $4.0 f_{c}$ and the minimum IL $0.75 \mathrm{~dB}$ within the passband, is designed on $0.254 \mathrm{~mm}$ PCB substrate with relative dielectric constant 2.2. As a result, the effective dimensions of the fabricated HPF are approximately $2.8 \mathrm{~mm}$ $\times 4.2 \mathrm{~mm}\left(\approx 0.062 \lambda_{g} \times 0.093 \lambda_{g}\right)$, where $\lambda_{g}$ is the guided wavelength at the $3 \mathrm{~dB}$ cutoff frequency $f_{c}$ in the substrate.

\section{Proposed Nonuniform CPW with Dual-Mode Resonator and HPF}

In Figure 1(a), a traditional short-circuited CPW resonator and its transmission-line equivalent circuit are shown. In general, the conventional uniform CPW with short-circuited structure only exhibits a single-mode resonant property, as shown in Table 1.

The input impedance of the equivalent transmission-line circuit shown in Figure 1(a) is calculated by the following equation:

$$
Z_{\text {in }}=j Z_{\text {con } 0} \tan \left(\theta_{\text {cono } 0}\right),
$$

where $Z_{\text {cono }}$ is the characteristic impedance of the conventional short-circuited $\mathrm{CPW}$ resonator and $\theta_{\text {cono }}$ is the electrical length of the resonator.

In order to obtain a wideband high-pass response within our concerning frequency range, a modified short-circuited CPW resonator with nonuniform stepped impedance structure at the bottom of the dielectric substrate is presented in our study and shown in Figure 1(b), which is used to create an ultrawide dual-mode resonant response. The transmission-line equivalent circuit of the proposed CPW is also given in Figure 1(b), which is composed of five-section stepped impedance units, corresponding to the different
TABLE 1: Comparison of transmission zeros (TZs) and resonant peaks for the conventional and proposed CPW resonator.

\begin{tabular}{lccc}
\hline Type of resonators & TZs $(\mathrm{GHz})$ & $f_{r 1}(\mathrm{GHz})$ & $f_{r 2}(\mathrm{GHz})$ \\
\hline Conventional CPW & 5.26 & 11.51 & N/A \\
Proposed CPW & 3.95 & 8.07 & 20.25 \\
\hline
\end{tabular}

characteristic impedances. Similarly, the input impedance of the equivalent transmission-line circuit shown in Figure 1(b) is calculated by the following series equations:

$$
\begin{aligned}
& Z_{\mathrm{in}, 1}=j Z_{0,1} \tan \left(\theta_{0,1}\right) \\
& Z_{\mathrm{in}, k}=Z_{0, k} \frac{Z_{\mathrm{in}, k-1}+j Z_{0, k} \tan \left(\theta_{0, k}\right)}{Z_{0, k}+j Z_{\mathrm{in}, k-1} \tan \left(\theta_{0, k}\right)}, \quad k=2,3,4,5,
\end{aligned}
$$

where $Z_{0, k}(k=1,2,3,4,5)$ is the characteristic impedance of the proposed CPW SIR and $\theta_{0, k}(k=1,2,3,4,5)$ is the corresponding electrical length of the proposed resonator.

Based on the following physical dimensions, that is, $w_{c 1}=$ $w_{c 2}=2.6 \mathrm{~mm}, l_{c 1}=1.6 \mathrm{~mm}, l_{c 2}=0.4 \mathrm{~mm}, w_{s 1}=0.8 \mathrm{~mm}$, $w_{s 2}=1.2 \mathrm{~mm}, l_{s 1}=0.5 \mathrm{~mm}, l_{s 2}=0.8 \mathrm{~mm}, l_{s 3}=0.4 \mathrm{~mm}$, and $s=0.1 \mathrm{~mm}$, the dual-mode resonant characteristic of proposed improved CPW has been examined in this paper. The inspected results, including the conventional short-circuited CPW with identical width $\left(w_{c}=w_{c 1}=w_{c 2}\right)$ and total length $\left(l_{c}=l_{c 1}+l_{c 2}+w_{s 1}+w_{s 2}\right)$ for a comparison with the proposed CPW, are also shown in Table 1. Obviously, the wide dualmode resonant property of proposed CPW has been clearly observed. The four critical geometrical variables $l_{s 1}, l_{s 2}, w_{s 1}$, and $w_{s 2}$ have been further investigated, and the dual-mode resonant responses from the weakly coupled excitation have been also examined in this design. The frequency ratios, from the dual-mode characteristics of the proposed resonator, have been exhibited in Figure 2, as the above-mentioned four 


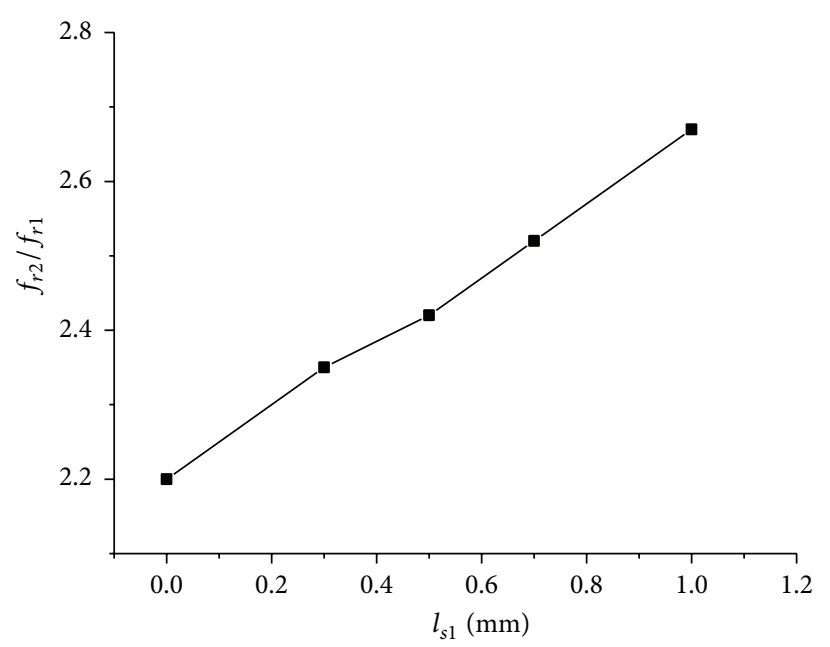

(a)

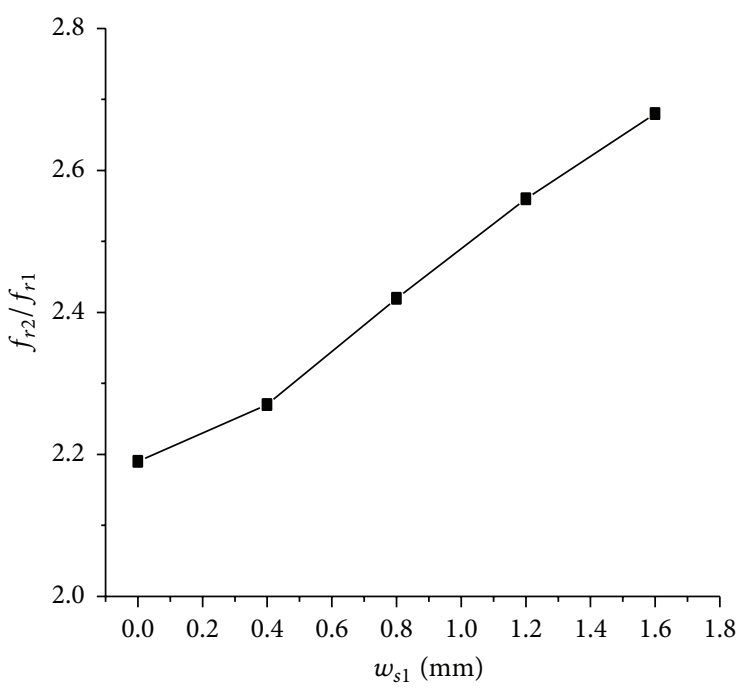

(c)

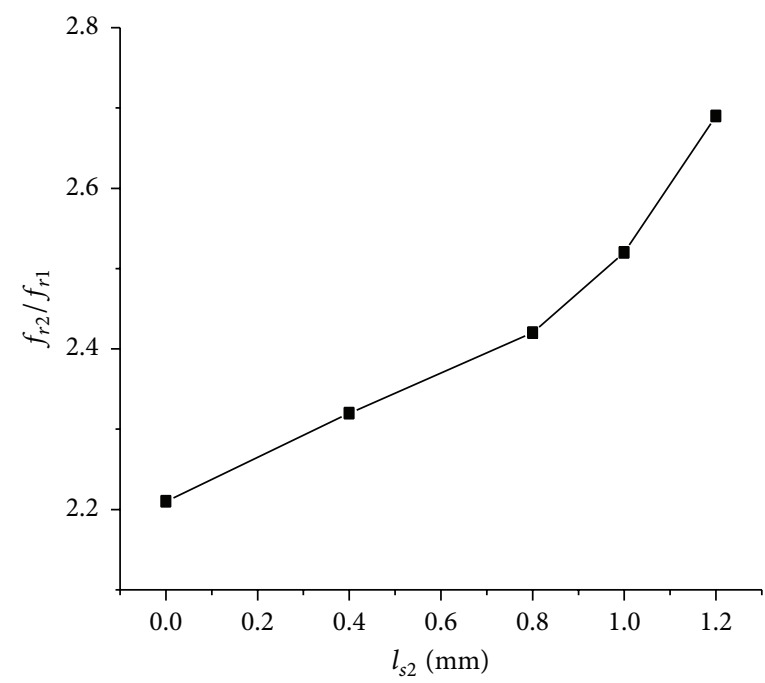

(b)

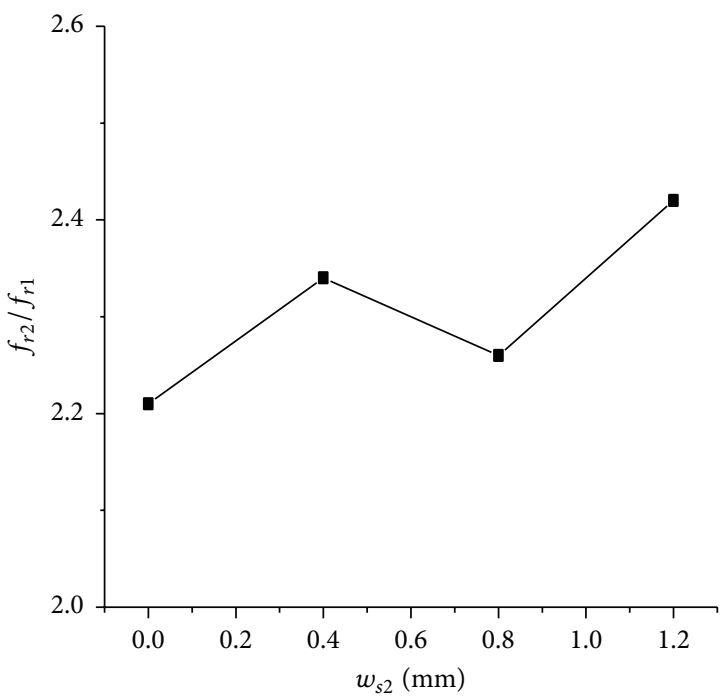

(d)

FIGURE 2: Dual-mode resonant frequency ratio of nonuniform CPW within the high-pass range for different parameters and dimensions (unit: mm). (a) $f_{r 2} / f_{r 1}$ varies against variable $l_{s 1}$, (b) $f_{r 2} / f_{r 1}$ varies against variable $l_{s 2}$, (c) $f_{r 2} / f_{r 1}$ varies against variable $w_{s 1}$, and (d) $f_{r 2} / f_{r 1}$ varies against variable $w_{s 2}$.

parameters vary. From the four subplots, when the other geometrical dimensions are kept unchanged, the frequency ratio $f_{r 2} / f_{r 1}$ will almost increase with the lengthening of the four variables. That is, the spacing between the dual-mode resonant frequencies (i.e., $f_{r 1}$ and $f_{r 2}$ ) will enlarge as these parameters increase. As a result, the passband width of the filter will be widened. In addition, the second resonant peak has not been nearly affected by the variable $l_{s 2}$; however, the first resonant peak will move from $8.6 \mathrm{GHz}$ down to the lower frequency $6.8 \mathrm{GHz}$ as the variable $l_{s 2}$ increases, when the variable $l_{s 1}=0.5 \mathrm{~mm}$. Particularly, the first resonant peak will disappear when $l_{s 2}$ is greater than $1.2 \mathrm{~mm}\left(l_{s 1}=0.5 \mathrm{~mm}, w_{s 1}=\right.$ $0.8 \mathrm{~mm}, w_{s 2}=1.2 \mathrm{~mm}$, and other physical dimensions are unchanged). Furthermore, there are two transmission zeros observed in the study, which are distributed at the lower and upper sides of two resonant peaks. This result also explains the reasons why the second transmission zero within the stopband of proposed HPF is located near $3.5 \mathrm{GHz}$, and there is a parasitic passband attenuation exceeding $23.0 \mathrm{GHz}$.

In order to obtain the high-pass frequency response, two identical top microstrip rectangular patches, shown in Figure 3(a), are separated with dimension $g_{1}$, which mainly contributes to the stopband attenuation at the lower frequency band. At the same time, the top microstrip spacing $g$, which is equivalent to lump capacitance with blocking direct current (DC) property, generates the transmission zero (TZ) near the DC operating point. Additionally, the coupled microstrip patches are in central alignment with the symmetric line of the CPW in the ground of the single substrate. Therefore, the proposed HPF does not use any viahole to the grounding plane, while the via-hole usually brings about fabricating difficulty. Finally, making use of the hybrid 


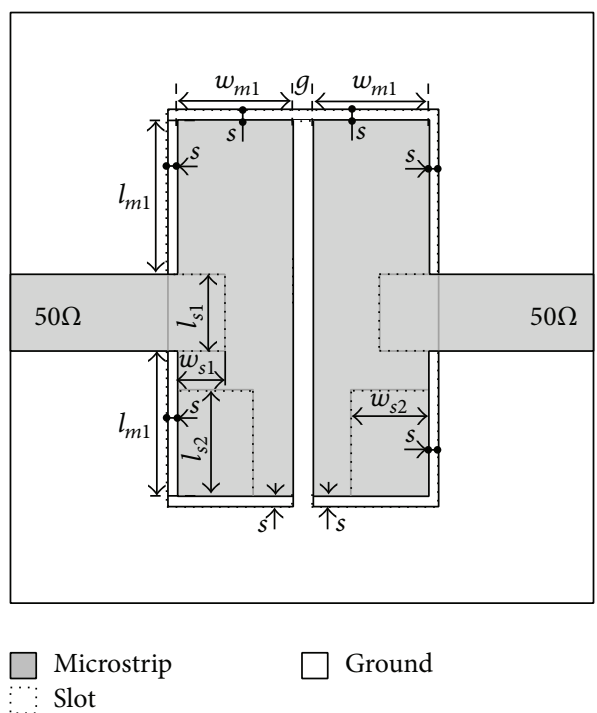

(a)

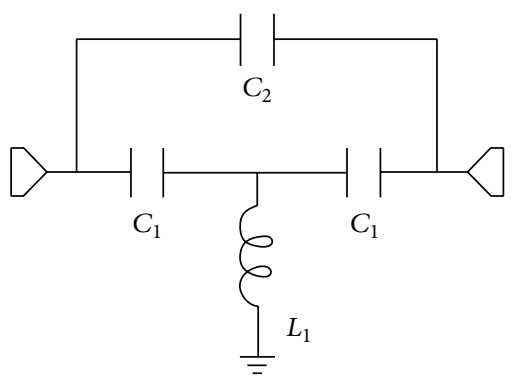

(b)

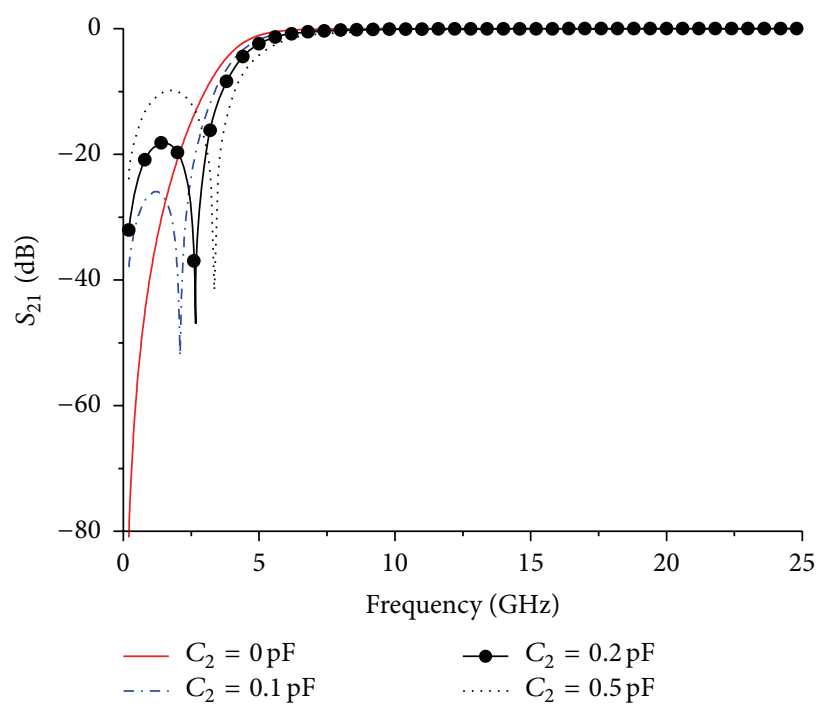

(c)

Figure 3: (a) Configuration of the proposed HPF, (b) its lumped-element equivalent circuit, and (c) circuit simulated results of the three-pole high-pass filter prototype for various values of the cross-coupled capacitance $C_{2}\left(C_{1}=0.649 \mathrm{pF} ; L_{1}=1.051 \mathrm{nH}\right)$.

broadside coupling between top microstrip patch and bottom dual-mode CPW resonator, an excellent high-pass response will be achieved.

To explain further the operational schematic of the designed HPF, a lumped-element equivalent circuit is given in Figure 3(b), which shows a three-pole high-pass response. The lumped filter will be adopted to generate a very wideband passband. Here, the series capacitances $C_{1}$ and the shunt inductance $L_{1}$ are selected to decide the cutoff frequency of the high-pass filter, and the cross-coupled capacitance $\mathrm{C}_{2}$ is introduced to create a transmission zero at the passband edge so that the filter selectivity at the stopband may be improved. Figure 3(c) shows the circuit simulated frequency response of this three-pole high-pass prototype for which the value of cross-coupled capacitance $C_{2}$ is adjusted to control the location of the created transmission zero. In Figure 3(b), the series capacitors $C_{1}$ represent the microstripto-CPW transition and the shunt inductor $L_{1}$ simulates the stepped impedance CPW short-circuited resonator, shown in Figure 1(b). At the same time, the cross-coupled capacitor $\mathrm{C}_{2}$ is equivalent to the coupling between the two adjacent microstrip patch resonators. In our design, the lumped parameters $C_{1}$ and $C_{2}$ can be extracted by the full-wave electromagnetic (EM) simulator, while the element $L_{1}$ is calculated by the input impedance of the SI CPW resonator, shown in (2a) and (2b).

In Figure 4, a comparison of the magnitude responses on the transmission and reflection loss between the conventional and proposed CPW resonators, based on the identical microstrip patch structure as depicted in Figure 3(a), is 


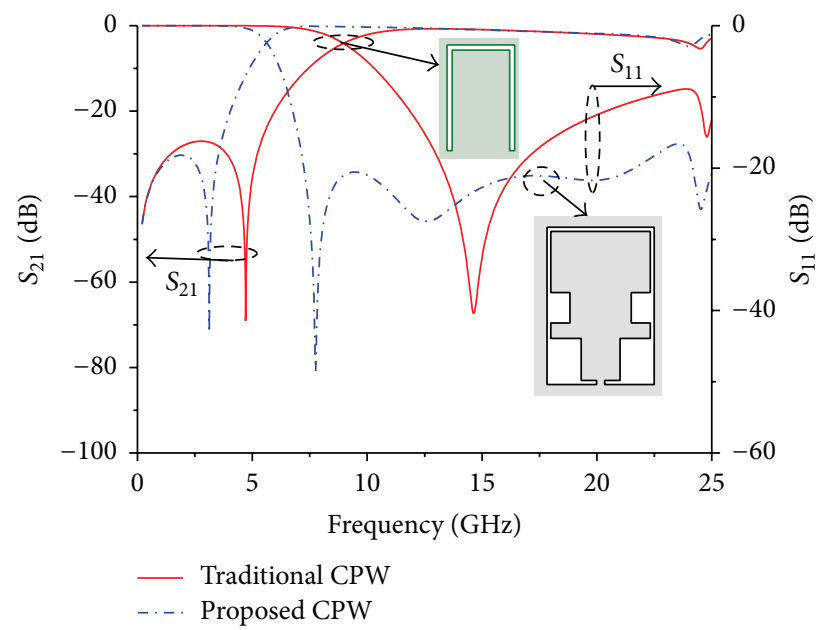

FIGURE 4: Comparison of frequency responses between the HPF using conventional CPW and the one using proposed CPW.

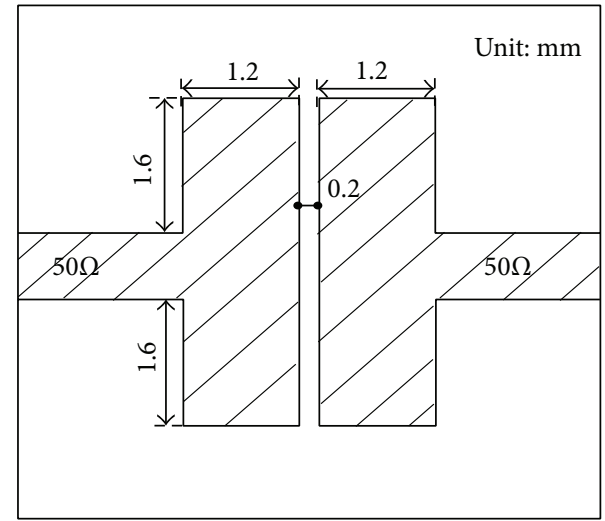

(a)

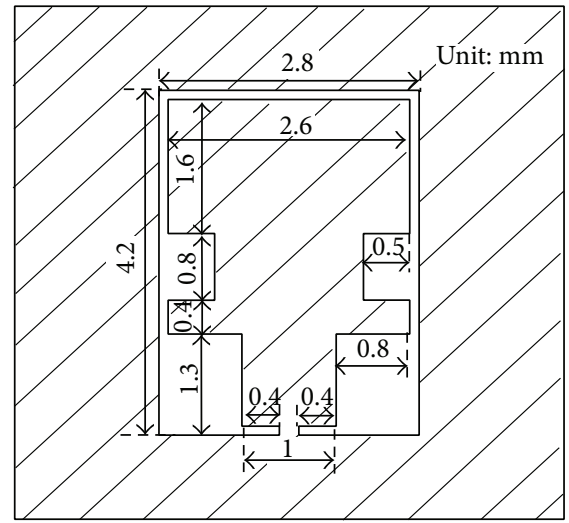

(b)

Figure 5: Implemented HPF layout and its dimensions. (a) Top coupled microstrip and (b) bottom nonuniform CPW.

shown. It is clearly seen that $S_{21}$ and $S_{11}$ of proposed HPF using nonuniform CPW resonator are better than the ones of conventional HPF using uniform resonator. In addition, the transmission zero of the proposed HPF is lower than that of the conventional one; therefore, its geometrical size is more compact than that of the latter.

After discussing the dual-mode characteristic of CPW resonator, explaining the operational scheme of the lumped equivalent circuit for the proposed HPF and investigating the important physical dimensions, the initial geometric parameters have been calculated by the above-described frequency response characteristic. Taking advantage of advanced optimum and simulation performances from the full-wave EM simulator HFSS, the final geometric sizes have been obtained. These complete dimensions of designed HPF have been shown in Figure 5. The dimensions of top microstrip patches are marked clearly in Figure 5(a), while the sizes of bottom $\mathrm{CPW}$ resonator are indicated explicitly in Figure 5(b).

\section{Experimental Results}

Based on the above-defined physical dimensions of the designed HPF shown in Figure 5, an advanced and miniaturized HPF has been simulated, fabricated, installed, and measured. The photograph of implemented filter is shown in Figure 6. The simulation and measurement have been accomplished using the commercially available EM simulation software HFSS and a vector network analyzer (VNA), respectively. Figure 7 (a) shows the simulated and measured insertion loss and return loss of the proposed filter. It can be seen that a good agreement between the simulated and measured results has been achieved, except that there is a little deviation at the lower stopband response of $\left|S_{21}\right|$, which might be mainly fabrication and installation errors for the dual-metal-plane layout. Seen from Figure 7(a), the measured $3 \mathrm{~dB}$ cutoff frequency is $f_{c}=5.78 \mathrm{GHz}$, and the high frequency (HF) passband range of $3 \mathrm{~dB}$ IL is up to $22.96 \mathrm{GHz}$, 


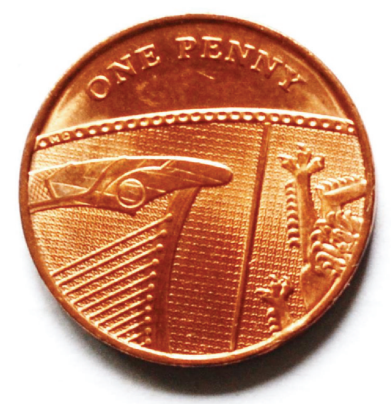

(a)

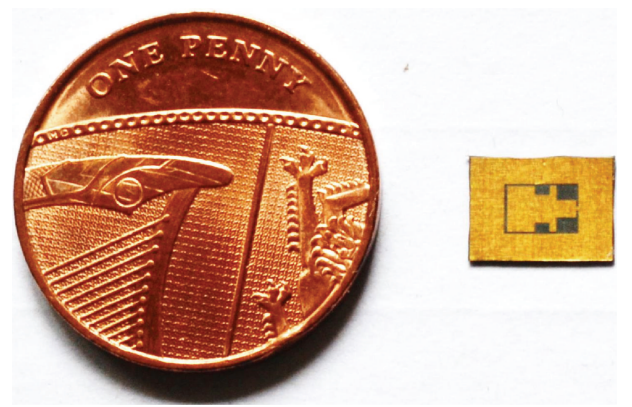

(b)

FIGURE 6: Photographs of fabricated HPF. (a) Top microstrip and (b) bottom modified CPW.

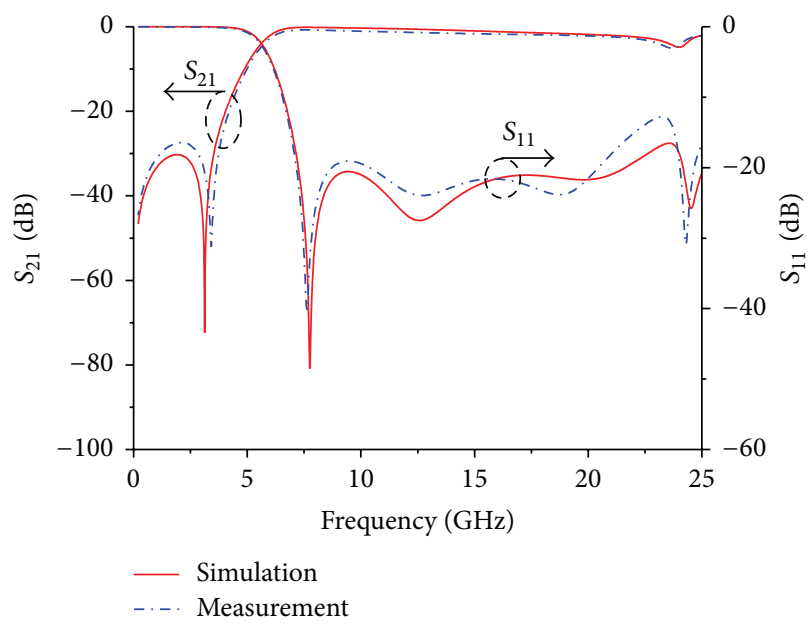

(a)

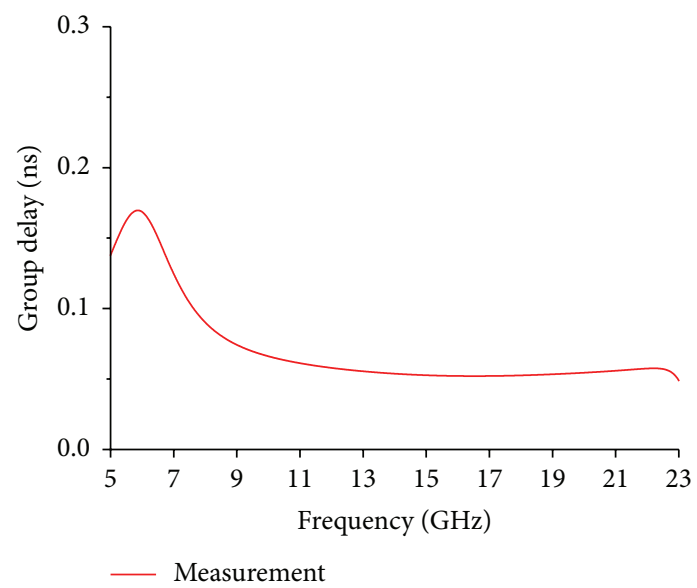

(b)

Figure 7: Simulated and measured results of the implemented HPF. (a) Magnitude responses and (b) measured group delay.

which exhibits the highest passband frequency approximately $4.0 f_{c}$. Within the passband, the measured minimum IL is $0.75 \mathrm{~dB}$ at $7.51 \mathrm{GHz}$. At the same time, there are three transmission poles observed within the passband, that is, $7.62,12.69$, and $18.73 \mathrm{GHz}$, respectively. The measured return loss within the high-pass range is better than $20 \mathrm{~dB}$ from $7.16 \mathrm{GHz}$ to $20.51 \mathrm{GHz}$. In addition, two transmission zeros have been obtained, which are near DC operating point and an attenuation $-52.1 \mathrm{~dB}$ at $3.42 \mathrm{GHz}$, respectively. From DC to $3.74 \mathrm{GHz}$, the measured transmission attenuation at the lower stopband is better than about $30 \mathrm{~dB}$. In Figure 7(b), the group delay (GD) of the fabricated HPF is also measured. Within the main passband range, the variation of GD is lower than $0.15 \mathrm{~ns}$ from $5.0 \mathrm{GHz}$ to $23.0 \mathrm{GHz}$. In addition, a comparison of measured results or other important parameters from our work and some typical references is listed in Table 2.

\section{Conclusion}

In this paper, a new and ultraminiature HPF based on a dual-mode CPW resonator with an ultrawide resonant response has been designed, manufactured, and measured. The wideband high-pass response has been implemented by the CPW resonator coupled broadside to the top microstrip patches. The fabricated HPF has exhibited an ultrawide passband filtering response of $3 \mathrm{~dB}$ IL up to about $4.0 f_{c}$, a good return loss better than $20 \mathrm{~dB}$ within the passband $7.16 \mathrm{GHz}$ to $20.51 \mathrm{GHz}$, and a good stopband attenuation characteristic with two transmission zeros. In addition, the proposed HPF has a very simple geometrical structure and circuit topology. Therefore, the filter is easily designed and implemented via the conventional PCB technology. The concept and approach to the HPF proposed in this paper could also be used to implement the ultra-wideband (UWB) bandpass filters (BPFs), which are based on the cascade of high- and low-pass filters.

\section{Competing Interests}

The authors declare that they have no competing interests.

\section{Acknowledgments}

This work was supported by the Natural Science Foundation of China (U1233103 and 61501091), the Central University Basic Research Funding (ZYGX2014J003), the Science and 
TABLE 2: Comparison of various HPFs in different works.

\begin{tabular}{lccccccc}
\hline Ref. & $\begin{array}{c}f_{c} / \mathrm{GHz} \\
\text { at } 3 \mathrm{~dB}\end{array}$ & $\begin{array}{c}\text { ILmin/dB in } \\
\text { the passband }\end{array}$ & $\begin{array}{c}\text { Sizes } \\
\left(\lambda_{g} \times \lambda_{g}\right)\end{array}$ & $f_{\text {pmax }} / f_{c}$ & $\begin{array}{c}\text { ILmin/dB in } \\
\text { the stopband }\end{array}$ & RL/dB in the passband & GD/ns in the passband \\
\hline$[4]$ & 1.74 & 0.33 & N/A & 4.5 & $>60$ & $\approx 10$ & N/A \\
{$[5]$} & 1.5 & 2.2 & 0.37 (length) & 2.0 & $>40$ & $\approx 10$ & N/A \\
{$[6]$} & 1.45 & 2.0 & N/A & 1.7 & $>30$ & N/A & N/A \\
{$[7]$} & 1.8 & 1.3 & $0.08 \times 0.09$ & 3.0 & $>40$ & $>7$ & N/A \\
{$[8]$} & 3.0 & N/A & $0.092 \times 0.185$ & 2.0 & $>40$ & $>15$ & $<0.15$ \\
This work & 5.78 & 0.75 & $0.062 \times 0.093$ & 4.0 & $>30$ & $>20$ & \\
\hline
\end{tabular}

Technology Support Plan of Sichuan Province (2015RZ0012), and Shanghai Aerospace Science and Technology Innovation Fund Project. H. Chen also thanks the Chinese Scholarship Council for the support.

\section{References}

[1] J.-S. Hong and M.-J. Lancaster, Microstrip Filters for RF/ Microwave Applications, Wiley, New York, NY, USA, 2001.

[2] C. Li, K.-Y. Liu, and F. Li, "Design of microstrip highpass filters with complementary split ring resonators," Electronics Letters, vol. 43 , no. 1 , pp. 35-36, 2007.

[3] J.-C. Liu, D.-S. Shu, B.-H. Zeng, and D.-C. Chang, "Improved equivalent circuits for complementary split-ring resonatorbased high-pass filter with C-shaped couplings," IET Microwaves, Antennas and Propagation, vol. 2, no. 6, pp. 622-626, 2008.

[4] J. Selga, G. Sisó, M. Gil, J. Bonache, and F. Martín, "Microwave circuit miniaturization with complementary spiral resonators: application to high-pass filters and dual-band components," Microwave and Optical Technology Letters, vol. 51, no. 11, pp. 2741-2745, 2009.

[5] J. Selga, M. Gil, F. Aznar, J. Bonache, and F. Martín, "Composite right-left-handed coplanar waveguides loaded with split ring resonators and their application to high-pass filters," IET Microwaves, Antennas \& Propagation, vol. 4, no. 7, pp. 822-827, 2010.

[6] N. Shoaib, I. Shoaib, and S. Shoaib, "A novel approach to transform an open circuit series stub to a short circuit shunt stub for the implementation of high-pass butterworth filter," Microwave and Optical Technology Letters, vol. 55, no. 3, pp. 497501, 2013.

[7] M. Durán-Sindreu, J. Bonache, and F. Martín, "Elliptic highpass filters with stepped impedance resonators in coplanar waveguide technology," Microwave and Optical Technology Letters, vol. 54, no. 4, pp. 1094-1097, 2012.

[8] H. Chen, P. Tang, K.-S. Chen, H.-F. Zhao, and H.-S. Zhong, "Wideband dual-mode bandpass filter using a modified righttriangular patch resonator overlapped with input/output DMS," Journal of Electromagnetic Waves and Applications, vol. 27, no. 11, pp. 1365-1371, 2013.

[9] J.-W. Baik, L. Zhu, and Y.-S. Kim, "Dual-mode dual-band bandpass filter using balun structure for single substrate configuration," IEEE Microwave and Wireless Components Letters, vol. 20, no. 11, pp. 613-615, 2010.

[10] H. Chen, H.-F. Zhao, K.-S. Chen, Y.-H. Wu, P. Tang, and H.S. Zhong, "A high selectivity quad-band bandpass filter based on hybrid-coupled microstrip/slotline quad-mode resonator,"
Journal of Electromagnetic Waves and Applications, vol. 27, no. 15, pp. 1902-1909, 2013.

[11] S. Luo and L. Zhu, "A novel dual-mode dual-band bandpass filter based on a single ring resonator," IEEE Microwave and Wireless Components Letters, vol. 19, no. 8, pp. 497-499, 2009.

[12] Y.-C. Chiou, C.-Y. Wu, and J.-T. Kuo, "New miniaturized dualmode dual-band ring resonator bandpass filter with microwave C-sections," IEEE Microwave and Wireless Components Letters, vol. 20, no. 2, pp. 67-69, 2010.

[13] H. Liu, J. Lei, J. Wan, Y. Wang, F. Yang, and S. Peng, "A miniaturized dual-mode bandpass filter using slot spurline technique," International Journal of Antennas and Propagation, vol. 2013, Article ID 853594, 6 pages, 2013.

[14] N. Thomson and J.-S. Hong, "Compact ultra-wideband microstrip/coplanar waveguide bandpass filter," IEEE Microwave and Wireless Components Letters, vol. 17, no. 3, pp. 184-186, 2007.

[15] T.-N. Kuo, C.-H. Wang, and C. H. Chen, "A compact ultrawideband bandpass filter based on split-mode resonator," IEEE Microwave and Wireless Components Letters, vol. 17, no. 12, pp. 852-854, 2007 


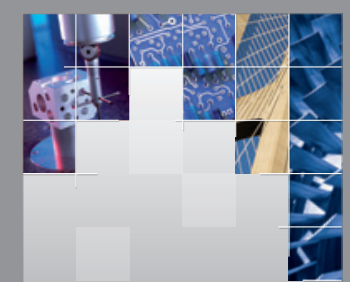

\section{Enfincering}
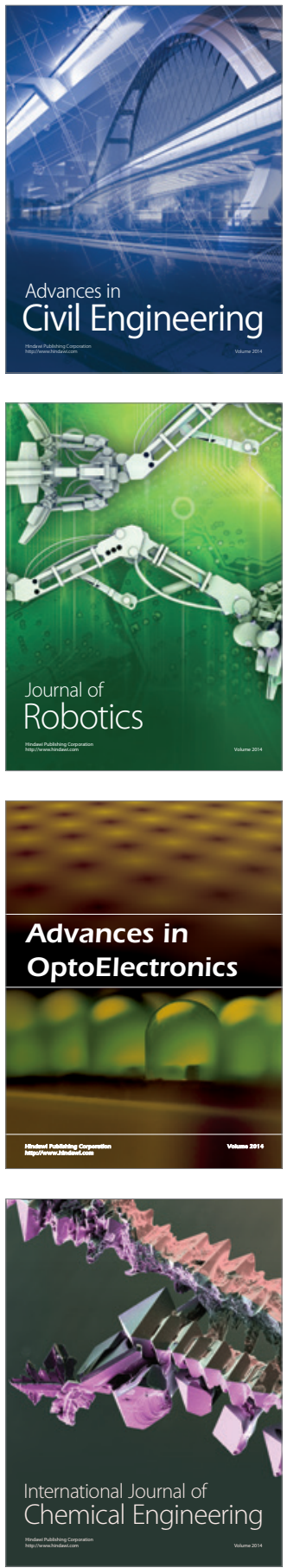

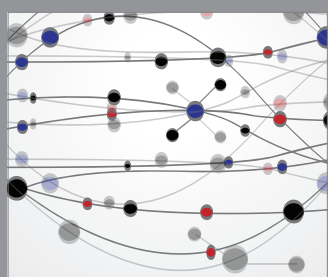

The Scientific World Journal

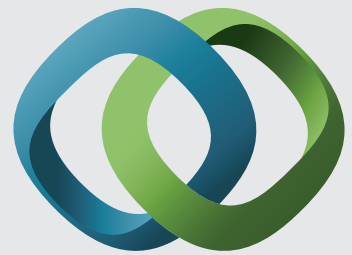

\section{Hindawi}

Submit your manuscripts at

http://www.hindawi.com
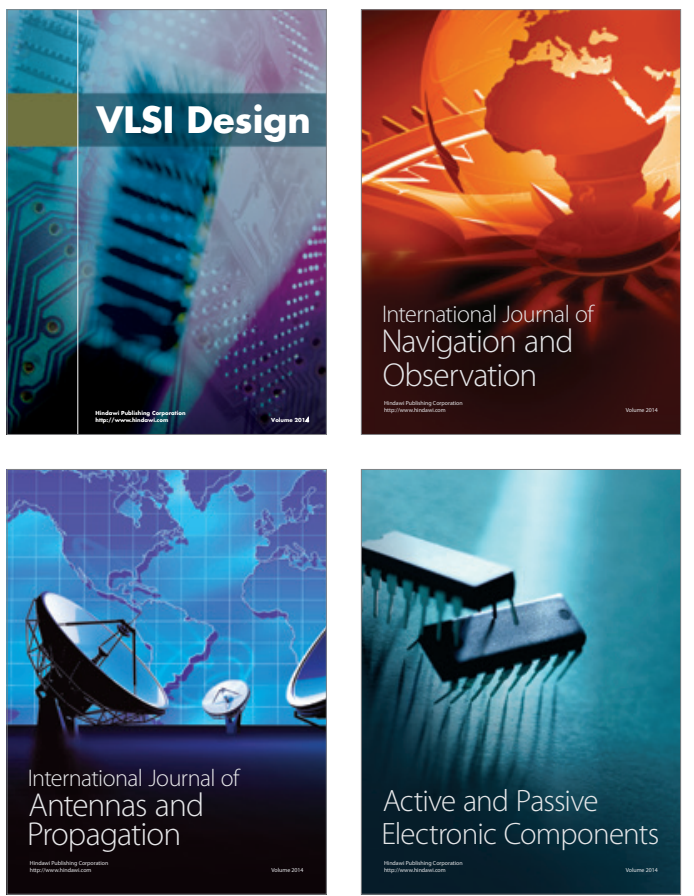
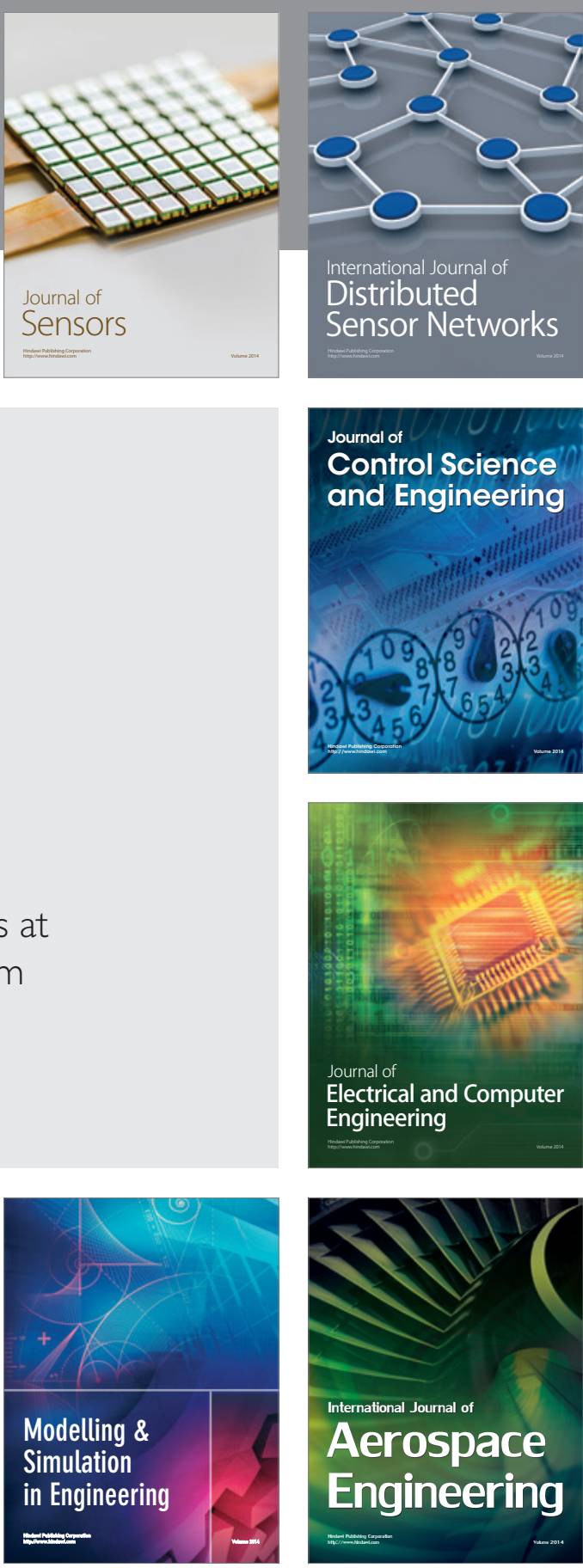

International Journal of

Distributed

Sensor Networks

Journal of

Control Science

and Engineering
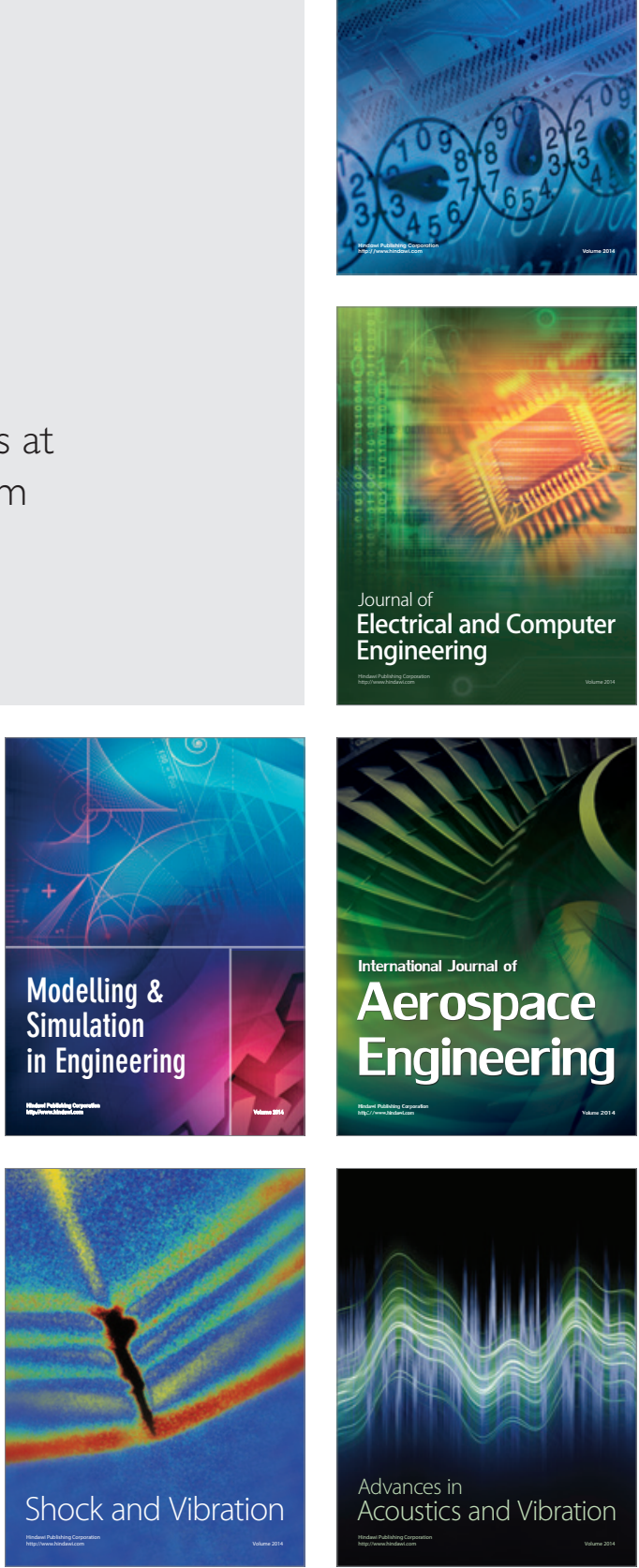